\title{
RF-driving of Acoustic-Optical Tunable Filters; design, realization and qualification of analog and digital modules for ESA
}

J. Vanhamel (1), D. Fussen (2), E. Dekemper (3), E. Van Lil (4), P. Leroux (5), E. Neefs (6), B. Van Opstal (7),

D. Pieroux (8), J. Maes (9), V. Voloshinov (10)

1 : Belgian Institute for Space Aeronomy, Ringlaan 3, 1180 Brussels - Belgium

Email: jurgen.vanhamel@aeronomie.be

2 : As (1) above, but E-mail: didier.fussen@aeronomie.be

3 : As (1) above, but E-mail: emmanuel.dekemper@aeronomie.be

4: ESAT - TELEMIC, Telecommunications and Microwaves KULeuven, Kasteelpark Arenberg 10, bus 244, 3001

Leuven - Belgium, E-mail: emmanuel.vanlil@esat.kuleuven.be

5: Technologycluster ESAT, Technologiecampus Geel, Kleinehoefstraat 4, 2440 Geel - Belgium

E-mail: paul.leroux@kuleuven.be

6 : As (1) above, but E-mail: eddy.neefs@aeronomie.be

7: As (1) above, but E-mail: bert.vanopstal@aeronomie.be

8: As (1) above, but E-mail: didier.pieroux@aeronomie.be

9: As (1) above, but E-mail: jeroen.maes@aeronomie.be

10: M.V. Lomonosov Moscow State University, Faculty of Physics, Leninskie Gory, Moscow 119991, Russia Email: volosh@phys.msu.ru

\begin{abstract}
The ALTIUS-instrument is a three-channel spectral imager, measuring in the ultraviolet (UV) (250 $\mathrm{nm}$ to $450 \mathrm{~nm}$ ), visible $(440 \mathrm{~nm}$ to $800 \mathrm{~nm}$ ) and near infrared (NIR) $(900 \mathrm{~nm}$ to $1800 \mathrm{~nm}$ ) wavelength domains, that is bound to fly aboard a PROBA-satellite. The ALTIUS-project (satellite and instrument) is developed under the supervision of ESA and with funding of Belgian Science Policy (BELSPO). The goal of the ALTIUS-instrument is to make hyper spectral images of the limb of the earth. To do this, the instrument will use different observation techniques such as "direct limb viewing", "stellar occultation" as well as "solar occultation". In each of ALTIUS' three channels an AOTF (Acousto-Optical Tunable Filter) will be used. The AOTF is used as a passband selector making it possible to scan fast and randomly through the spectral domain. The AOTF is the key element of the instrument's front end optics. As spectral slices of the incoming light are rhythmically passed to a two dimensional detection system, ALTIUS is capable of generating hyperspectral images of the observed scene.
\end{abstract}

For each of the three AOTFs an RF-generator will be developed, with dedicated requirements with respect to resolution, sensitivity, frequency range, electrical and optical performance. The electronics will be designed and manufactured to survive several years in a space environment. It needs to pass a suite of environmental tests such as thermal-vacuum, vibration, radiation, shock and EMC. The choice of electronic components, the PCBdesign and manufacturing needs to be in accordance with "space qualified" standards.

For the AOTF RF-generator the use of an analog Hilbert transform is proposed as a possible solution. By combining a space qualified fixed oscillator with a space qualified FPGA, the desired frequency ranges can be reached for the three channels. Inside the FPGA a DDS (Direct Digital Synthesizer) is integrated. The DDS in combination with a space qualified DAC (Digital to Analog Convertor) produces a sine wave of 0 to $30 \mathrm{MHz}$.

This master signal is combined with a fixed $90 \mathrm{MHz}$ oscillator to produce a driving frequency between 60 and 120 $\mathrm{MHz}$ for the visible channel.

For the NIR channel the use a fixed space qualified oscillator of $45 \mathrm{MHz}$ in combination with the master output of the DDS-DAC (here running at a frequency between 0 and $15 \mathrm{MHz}$ ) fulfills the requirements. This combination leads to a driving frequency between 30 and $60 \mathrm{MHz}$.

Finally for the UV-channel switchable fixed space qualified oscillators of $165 \mathrm{MHz}$ and $235 \mathrm{MHz}$ are used in combination with the DDS-DAC (here running at a frequency between 0 and $35 \mathrm{MHz}$ ). By combining these two devices a driving frequency between 130 and $270 \mathrm{MHz}$ can be reached. The advantage of using a switchable oscillator is that more than one octave can be covered. 
The conceptual design of this approach has already been performed for the three channels (i.e. in the three frequency ranges) and has already been simulated, prototyped and tested. It is proven that the requirements put forward for the RF chains are achievable. Currently the unwanted spectral components in the RF-output are -30 $d B c$. Further research is carried out to improve the spectral purity of the produced sine waves

To reach the desired power levels to drive the AOTFs the above described RF-generators will be combined with an RF-driver/amplifier. As for the RF-generator, also during the RF-amplifier design the focus will be on low power consumption, limited volume and survivability in space. 